\title{
Cuantificación del carbono orgánico y materia orgánica en suelos no rizosféricos o cubiertos por Avicennia germinans (L.) y Conocarpus erectus (L.) emplazados en Boca de Uchire, laguna de Unare, Estado de Anzoátegui, Venezuela
}

Quantification of organic carbon and organic matter in nonrhizospheric soils and covered by Avicennia germinans (L.) and Conocarpus erectus (L.) located in Boca de Uchire, Unare lagoon, State of Anzoátegui, Venezuela

Franklin Núñez-Ravelo ${ }^{1}$

Universidad Pedagógica Experimental Libertador, Venezuela

María Ugas-Pérez ${ }^{2}$

Universidad Pedagógica Experimental Libertador, Venezuela Rossany Calderón-Castellanos ${ }^{3}$

Universidad Pedagógica Experimental Libertador, Venezuela Ferney Rivas-Meriño ${ }^{4}$

Universidad Pedagógica Experimental Libertador, Venezuela

1 Profesor en Geografía, Magister en Geografía, mención Geografía Física, Doctor en Educación Ambiental. Universidad Pedagógica Experimental Libertador-IPC. Correo electrónico: franklingeove@hotmail.com (iD) https://orcid.org/0000-0001-5501-3085

2 Profesora en Geografía, Magister en Geografía, mención Geografía Física. Universidad Pedagógica Experimental Libertador-IPC. Correo electrónico: mariaelenaugas_88@hotmail.com

(iD) https://orcid.org/0000-0003-4798-8805

3 Profesora en Geografía, Universidad Pedagógica Experimental Libertador-IPC. Correo electrónico: marietcalderon19@gmail.com (D) https://orcid.org/0000-0002-2795-0183

4 Profesor en Geografía. Magister en Educación Ambiental. Universidad Pedagógica Experimental Libertador-IPC. Correo electrónico: fernneyrivas@gmail.com (D) https://orcid.org/0000-0001-6946-3992 
Quantification of organic carbon and organic matter in non-rhizospheric soils and covered by Avicennia germinans (L.) and Conocarpus erectus (L.) located in Boca de Uchire, Unare lagoon,

State of Anzoátegui, Venezuela.

\title{
Resumen
}

El propósito fue evidenciar la diferencia significativa en el contenido de carbono orgánico (C) y materia orgánica (MO) en tres grupos de suelos: no rizosférico $(n r)$, con presencia de Avicennia germinans $(\mathrm{Ag})$ y Conocarpus erectus $(\mathrm{Ce})$. Para este fin, se colectaron 54 muestras de suelo superficial (0-20 cm) en el sector Boca de Uchire, distribuidas en: 18 de $n r, 18$ de $\mathrm{Ag}$ y 18 de $\mathrm{Ce}$. Se determinó el contenido de $\mathrm{C}$ a partir de la oxidación por dicromato de potasio, así como el \% MO mediante el factor convencional de Bemmelen. Los datos obtenidos se trataron mediante análisis descriptivo, ANOVA y prueba de Tukey. Los resultados evidencian diferencias en los tres grupos de muestras para las variables en estudio, el C presentó valores promedios de $\mathrm{nr}=0,04 \% ; A g=0,08 \%$ y $C e=0,13 \%$, mientras que la MO se estimó en promedios de $\mathrm{nr}=0,07 \% ; A g=0,15 \%$ y $C e=0,23 \%$. Se reconoce que entre los grupos estudiados, el $\mathrm{Ce}$ presentan la mayor potencialidad forestal al aportar $2,73 \mathrm{Mg} \mathrm{C}^{-1}$ al suelo.

Palabras clave: almacenamiento de carbono, captura de carbono, manglares, potencialidad forestal.

\begin{abstract}
The purpose of the present study was to evidence the significant difference in the content of soil organic carbon (SOC) and soil organic matter (SOM) in three groups, namely: non-rhizospheric $(\mathrm{nr})$, Avicennia germinans-covered (Ag) and Conocarpus erectus-covered (Ce). For this purpose, 54 surface soil samples (0-20 cm depth) were collected in the Boca de Uchire sector according to the following distribution: $18 \mathrm{nr}, 18 \mathrm{Ag}$ and $18 \mathrm{Ce}$. SOC content was determined by potassium dichromate wet-oxidation and percentage SOM calculated using the conventional van Bemmelen conversion factor $(=1.724)$. Obtained data was treated by descriptive statistics analysis of ANOVA and Tukey's test. The results show statistically significant differences among the three sample groups for the variables under study. SOC presented average values of $\mathrm{nr}=0.04 \%, \mathrm{Ag}=0.08 \%$ and $\mathrm{Ce}=0.13 \%$; average $\mathrm{SOM}$ was estimated as $\mathrm{nr}=0.07 \% ; \mathrm{Ag}=0.15 \%$ and $\mathrm{Ce}=0.23 \%$. Ce is recognized as presenting the greatest forest potentiality by stocking capacity $2.73 \mathrm{Mg} \mathrm{C}^{-1} \mathrm{ha}^{-1}$ to the soil. Keywords: Carbon storage; Carbon sequestration; Mangroves; Forest potential.
\end{abstract}

\section{Introducción}

La Materia Orgánica (MO) así como el Carbono Orgánico (CO), constituyen dos de las propiedades bioquímicas del suelo de mayor importancia, ya que suelen ser considerados como elementos incidentes en la calidad edáfica. En efecto, ambos componentes suelen incidir favorablemente en otras propiedades del suelo, considerando que: aumenta la capacidad de intercambio catiónico, contribuye con la neutralización del $\mathrm{pH}$, favorece la retención de humedad, mejora la estabilidad de suelos arcillosos al ayudar a aglutinar las partículas para formar agregados, fomenta la actividad microbiológica, favorece la liberación de nutrientes, entre otros atributos (Ibáñez, 2006; Martínez, et al. 2008; García et al., 2011; Sainz et al. 2011; Organización de las Naciones Unidas para la Alimentación y la Agricultura, FAO, 2017) 
La MO como fracción orgánica del suelo, proveniente de los residuos de plantas y animales que son incorporados a su estructura, luego de su descomposición asociada a la acción microbiana, se presenta de color pardo o negruzco, y constituye el 5\% del volumen en un suelo ideal (Robinson, 1967; Navarro et al., 1995; Casanova, 2005) o humus (Velasco, 1989; Gros y Domínguez, 1992; Ibáñez, 2006).

De este material orgánico, una parte es incorporada al suelo y otra es liberada en forma de $\mathrm{CO}_{2}$. En efecto, la oxidación de la $\mathrm{MO}$ genera la transformación del carbono que contiene, de la forma orgánica a la inorgánica, generando la producción de una fracción de $\mathrm{CO}_{2}$ que es emitido a la atmósfera (Alef, 1995; Peris, 2013).

De acuerdo con Swift (2001), la mayor reserva de C en el planeta Tierra, se encuentra distribuido en los suelos del mundo, siendo este superior 2.1 veces más que en la atmósfera, y aproximadamente 2.7 veces más que en las reservas biótica constituidas por las plantas en la tierra.

La Organización de las Naciones Unidas para la Alimentación y la Agricultura (FAO, 2001), refirió que, en condición aeróbica, una parte importante del carbono que ingresa al suelo (55 $\mathrm{Pg} \mathrm{C}$ año ${ }^{-1}$ a nivel global) es lábil y se mineraliza rápidamente, mientras que una menor proporción, se acumula a modo de humus estable $\left(0.4 \mathrm{Pg} \mathrm{C}\right.$ año $\left.^{-1}\right)$.

De acuerdo con Duhne (2011), los manglares tienen una alta capacidad para almacenar grandes cantidades de carbono, reseñando que "el carbono que se encontró en los sedimentos de los manglares fue en promedio cinco veces mayor que el de los bosques terrestres, tanto templados como tropicales" (p.5), lo cual puede estar generado en primera instancia por los suelos en los que crecen, los cuales son muy rico en materia orgánica, aunado al complejo sistema de raíces que detiene la fuerza de las olas, lo que favorece la sedimentación. Estas condiciones propician en el suelo, la escasez de oxígeno que reduce la velocidad de descomposición, lo que permite que el carbono se acumule.

Según Duhne (2011) “en los manglares se encuentra más carbono en el suelo que en la combinación de biomasa y suelo en los bosques tropicales" (p. 5).

En tal sentido, la Organización de las Naciones Unidas para la Alimentación y la Agricultura (2017), advirtió que "la absorción o secuestro 
de carbono por parte del manglar contribuye a reducir los gases de efecto invernadero que generan el cambio climático global" (p.153).

Ahora bien, en atención a lo planteado por Segura et al. (2004), se reconoce que el tipo de vegetación constituye uno de los factores determinante para el aporte del CO en los suelos, de allí que el contenido cambia con el tipo de vegetación, el tipo de material parental y los factores topográfico del área.

Enmarcados en esta premisa, la presente investigación tuvo como propósito identificar posibles variaciones significativas en el contenido de $\mathrm{CO}$ y $\mathrm{MO}$ en tres grupos de suelos (no rizosférico, y, con presencia de en superficie por Avicennia germinans y Conocarpus erectus) en el sector Boca de Uchire, Laguna de Unare, Venezuela.

\section{Área de Estudio}

En el caso particular de los manglares en Venezuela, estos colonizan una extensión discontinua de aproximadamente $1100 \mathrm{~km}$ de la línea de costa, en una ocupación aproximada del 29\%. Los bosques se caracterizan por la presencia de distintas especies, entre las que destacan: Rhizophora mangle, Avicennia germinans, Laguncularia racemosa y Conocarpus erectus (Pannier y Pannier, 1989).

Estos han sido objeto de diversas investigaciones, orientadas fundamentalmente a estudiar: su estructura (De Olivares, 1988; Cumana et al. 2000; Medina y Barboza 2003; Barboza et al. 2006; Del Mónaco et al. 2010 y López et al. 2011; Núñez y Ugas, 2018), así como, la actividad microbiana y almacenamiento de carbono (Sánchez et al. 2010; Rodríguez y Gómez 2016, Núñez y Ugas, 2019). No obstante, si bien se ha reportado el contenido de $\mathrm{MO}$ y $\mathrm{C}$ en zonas de manglar, es necesario contribuir con el estudio de las potencialidades que tienen algunas especies en el aporte de estos elementos al suelo que ocupan.

En este sentido, la investigación se desarrolló en el sector de Boca de Uchire, localizado entre $\operatorname{los} 10^{\circ} 07^{\prime} 50^{\prime \prime}-10^{\circ} 08^{\prime} 00^{\prime \prime} \mathrm{N}$ y $65^{\circ} 24^{\prime} 28^{\prime \prime}-$ $65^{\circ} 24^{\prime} 47^{\prime \prime} \mathrm{O}$, en la costa noroeste del estado Anzoátegui en Venezuela, específicamente al extremo oeste del cordón litoral que limita al norte a la Laguna de Unare (Figura 1). 
Cuantificación del carbono orgánico y materia orgánica en suelos no rizosféricos o cubiertos por Avicennia germinans (L.) y Conocarpus erectus (L.) emplazados en Boca de Uchire, laguna de Unare,

Estado de Anzoátegui, Venezuela.

De acuerdo con Roa (1990), el análisis de sedimentos permitió establecer la edad radiométrica de la laguna por medio del C14, indicando que se ubica entre 3940 y 2710 años atrás.

Se trata de un área de aproximadamente 14.33 ha, con altitudes que no sobrepasan los 5 metros en relación con el nivel medio del mar, limitando al norte con el Mar Caribe, por el sur y oeste con una serie de colinas que constituyen las últimas estribaciones de la cadena litoral del Sistema Montañoso, mientras que al este entra en contacto con la Laguna de Unare, constituyendo el extremo oeste de la barra arenosa que la separa del mar.

Figura 1. Mapa de localización del área de estudio

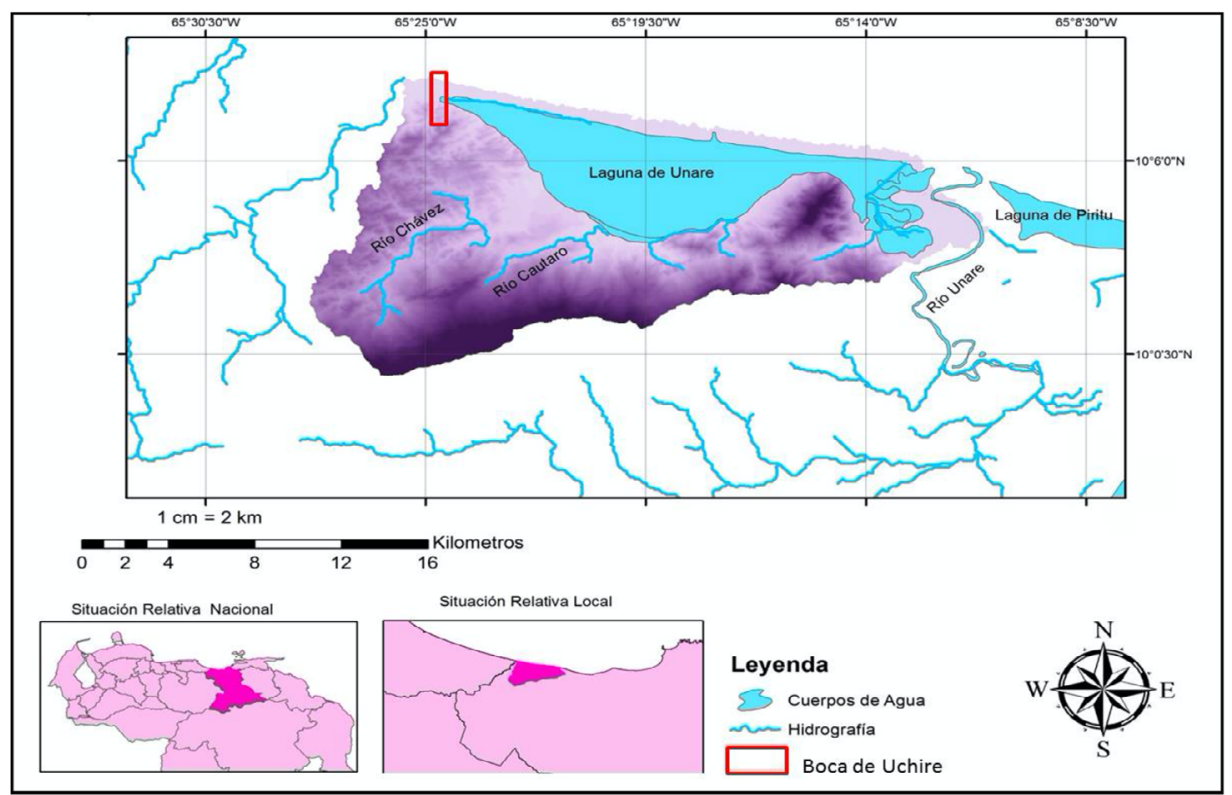

Fuente: Elaboración propia.

Tal altitud, la ubica en el piso megatérmico o tropical, con temperaturas elevadas $\left(>25^{\circ} \mathrm{C}\right)$ durante todo el año. Las crecientes precipitaciones, en sentido noroeste-suroeste, registran montos anuales que alcanzan 1400 $\mathrm{mm}$, concentradas en un marcado período de lluvias de 7 meses, comprendidos entre mayo y noviembre (Rodríguez y González, 2001), confiriéndole la condición de clima tropical estacional. 
El comportamiento de las precipitaciones, obedece al engolfamiento de los vientos alisios del NE, influenciados por las últimas estribaciones de la cadena litoral del Sistema montañoso del Caribe, en la subregión de Barlovento, entre Cabo Codera y Boca de Uchire (Vivas, 2012).

La estacionalidad de las precipitaciones incide en las condiciones morfodinámicas de la laguna. En efecto, Ojeda (2004), refiere que esta experimenta durante el período seco, una reducción de su extensión, pasando de $47.5 \mathrm{~km}^{2}$ hasta $44 \mathrm{~km}^{2}$, siendo más notable hacia sus bordes, ya que las aguas llegan a retirarse hasta $2 \mathrm{~km}$ de la barra que las separa del mar.

Adicionalmente, durante el período seco, los tres grau que presenta el cordón litoral (Unare, La Mora y Nueva), suelen cerrarse, como consecuencia de la reducción de los caudales de las quebradas que vierten sus aguas hacia el sector, y la sedimentación marina en el borde costero (Suarez, 1991; Rodríguez et al., 2009). Esto impide el flujo de agua entre el mar y la laguna, generando estancamiento de las aguas lagunares (Sebastiani et al., 2007).

Los ríos Cautaro, Chávez y Unare, constituyen las principales redes de drenaje hacia la laguna, generando su mayor descarga durante el período de precipitación, específicamente entre los meses de julio a noviembre, cuando drenan aproximadamente el $90 \%$ de su volumen de escurrimiento anual (Mattia y Méndez, 2003), confiriendo al agua una condición hiposalina (Senior y Ocando, s/f).

La baja salinidad en el agua de la laguna constituye un indicador del predominio del aporte fluvial sobre el marino en el sector. En efecto, de acuerdo con Méndez (2007), la variación de las mareas ha sido poco estudiada en la costa de Venezuela, sin embargo, se reconoce que en general, estas suelen ser en el orden de 30 a $40 \mathrm{~cm}$, con corrientes periódicas cuyos ciclos se completan en 24 horas, y su dirección se mantiene en un determinado sentido durante la mitad del período, para luego cambiar en sentido opuesto durante el resto del ciclo.

Lara et al. (1997), advierten que al no existir corrientes de mareas suficientemente fuertes en la costa de Unare, se reducen las condiciones que favorecen que los grau se mantengan abiertos durante el período de sequía.

Elizalde et al. (2007), afirman que en las zonas litorales próximas a la desembocadura del río Unare, predominan sedimentos arcillosos de origen marino, de edad reciente y ácidos. Las sales de origen marino acumuladas 
en el suelo se lixivian durante el período lluvioso, pero tienden a ascender a la superficie con las aguas capilares durante el período seco, clasificándolos en el orden Vertisols ${ }^{5}$, suborden Aquerts ${ }^{6}$, gran grupo Endoaquerts ${ }^{7}$.

Rodríguez et al. (2009), afirman que los sedimentos que constituyen la laguna son principalmente arcillosos, incrementando el porcentaje de arena en las zonas cercanas a la barra litoral (Boca Nueva y Mora) y la desembocadura del río Unare (Caño Norte).

Núñez y Ugas (2018), refieren para el sector en estudio, con base al análisis de frecuencia de clases texturales del suelo según la especie de manglar colonizante, que: los individuos de la especie Avicennia germinans crecen en $74.07 \%$ en suelos franco-arenosos, $14.81 \%$ en arenosos y 11.11 $\%$ en areno-francoso, mientras que los especímenes de Conocarpus erectus se desarrollan en un $42.86 \%$ sobre un sustrato areno-francoso, $28.57 \%$ en franco-arenoso y $14.29 \%$ en superficies arenosas o arcillo-arenosas.

Se estima que el origen de los manglares en este sector, se ubica en la década de los 70 del siglo XX, ya que según Roa (1990), este se desplazó del litoral, por acción del oleaje hacia la zona más internas de la laguna.

De acuerdo con Núñez y Ugas (2018), en la actualidad no se conoce la densidad del manglar en la zona, reconociendo su presencia hacia la zona norte y oeste de la laguna, desarrollados en parches de bosques abiertos o en algunos casos, en cobertura dispersa, tratándose de un manglar joven, con poco menos de 5 décadas de colonización (Figura 2).

\section{Marco Metodológico}

La investigación, en atención a su propósito, se desarrolló en tres fases: (a) trabajo de campo para recoger muestras, (b) análisis de laboratorio y (c) procesamiento estadístico de datos.

\section{Trabajo de Campo}

El trabajo de campo, se llevó a cabo entre el 12 y 14 de mayo de 2018, en el inicio de la estación lluviosa para la localidad, esto considerando que

5 Vertisols: suelos minerales arcillosos, se agrietan profundamente durante la época seca, y se expande en la época húmeda. Presentan superficies pulidas, producto de la fricción de grandes terrones en forma de cuña. (United States Department of Agriculture, 1999)

6 Aquerts: suborden del suelo Vertisols que se encuentran saturados de agua y sin oxígeno, al menos el tiempo suficiente como para afectar la actividad biológica de las especies vegetales no adaptadas a las condiciones de anegamiento. Típico de llanuras bajas. (United States Department of Agriculture, 1999)

7 Endoaquerts: presentan el nivel freático todo el año muy próximo a la superficie, por lo que generalmente están húmedos de abajo hacia arriba. (United States Department of Agriculture, 1999) 
Quantification of organic carbon and organic matter in non-rhizospheric soils and covered by Avicennia germinans (L.) and Conocarpus erectus (L.) located in Boca de Uchire, Unare lagoon, State of Anzoátegui, Venezuela.

las precipitaciones favorecen la humedad edáfica y "un porcentaje de humedad más alto favorece la actividad de los microorganismos en el suelo" (Ramos y Zuñiga, 2008, p. 125), los cuales resultan necesarios para la transformación de la hojarasca y otros residuos orgánicos, en MO y CO, que será incorporado a la estructura del suelo.

\section{Figura 2. Manglares en la Laguna de Unare}

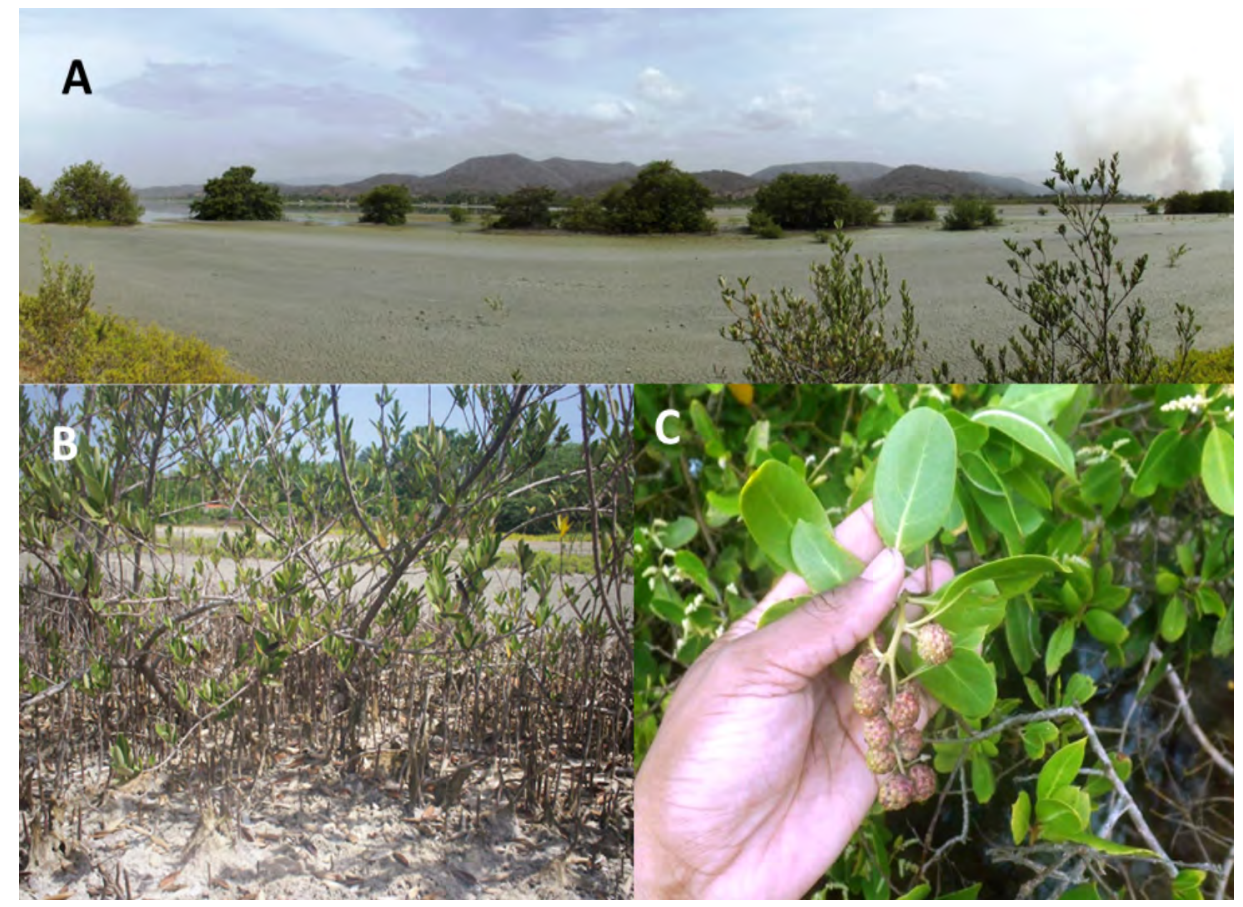

Nótese en los detalles: "A" en el centro de la imagen, el bosque con presencia de Avicennia germinans en cobertura dispersa, hacia el extremo inferior-derecho de la imagen el dosel de un individuo de la especie Conocarpus erectus, y al fondo, las últimas estribaciones de la Serranía del Interior del Sistema Montañoso; "B" individuo de Avicennia germinans con sus características neumorrizas sobre la superficie del suelo, y "C" individuo de Conocarpus erectus con sus típicos frutos en ramillete con drupas escamiforme de color marrón grisáceo, y hojas elípticas de base decurrente.

Fuente: Tomada por F. Núñez-Ravelo, 2018

Durante la ejecución de esta fase, se realizó la observación general del área, a fin de reconocer patrones de cobertura del manglar, caracterizándolo como un bosque mixto de Avicennia germinans y Conocarpus erectus, así como, identificar la accesibilidad y homogeneidad del terreno, 
procurando las áreas que visiblemente presentaran el menor impacto asociado a la acción antropogénica.

Por tratarse de un área en donde los manglares crecen en una cobertura abierta o sin contacto entre el dosel de los individuos, en campo se procedió a la colecta de suelo superficial ( $0-20 \mathrm{~cm}$ de profundidad), justificado en la imposibilidad técnica de recolectar muestras a mayor profundidad.

Se seleccionaron tres parcelas de 50×20 metros: (a) las dos primeras con presencia de Avicennia germinans, o Conocarpus erectus, colectando en cada caso, las muestra de suelo en el área más próxima a la base de los individuos arbóreos; y (b) la última parcela, en la zona desprovista de manglar, distanciada de las parcelas anteriormente referidas, por una distancia de 5 metros.

Bajo el criterio antes descrito, se colectaron 54 muestras de suelo, distribuidas en: (a) 18 muestras de suelo con presencia de Avicennia germinans, (b) 18 muestras de suelo con presencia de Conocarpus erectus, y (c) 18 muestras de suelo desprovisto de manglar.

Las muestras fueron resguardadas en bolsas de polietileno denso, identificadas con etiqueta contentiva de la siguiente información: clave única de identificación, coordenadas del punto de muestreo, fecha y hora de la colecta.

\section{Análisis de laboratorio}

Determinación del Carbono en el suelo

La determinación del C se llevó a cabo siguiendo el método propuesto por Walkley y Black (citado en Toledo, 2008). Previo a la determinación, se dispuso de los reactivos: (1) solución de dicromato de potasio al $8 \%$ y (2) ácido sulfúrico concentrado al $96 \%$.

Para la preparación de la curva de calibración de dextrosa para $\mathrm{CO}$, inicialmente se preparó la solución madre de concentración $50 \mathrm{mg} \mathrm{C} \mathrm{mL}$, para la cual se disolvió $13.76 \mathrm{~g}$ de dextrosa en $100 \mathrm{~mL}$ de agua destilada. A partir de la solución anterior, se generaron los patrones de concentración: $1.25 ; 2.5 ; 3.75 ; 5$ y $6.25 \mathrm{mg} \mathrm{C} \mathrm{mL.}$

Posteriormente, de cada una de las soluciones patrones, se extrajo 1 $\mathrm{mL}$ y se vertió en los respectivos tubos de ensayo con capacidad 15 a 20 $\mathrm{mL}$, luego a cada tubo se le agregó $2 \mathrm{~mL}$ de Dicromato de Potasio y $3 \mathrm{~mL}$ de Ácido Sulfúrico, se dejó enfriar por diez minutos y se le añadió $10 \mathrm{~mL}$ de $\mathrm{H}_{2} \mathrm{O}$ destilada, se agitó en el vortex y se centrifugó durante 10 minutos. 
De las soluciones centrifugadas, se extrajo en cada caso con una micropipeta, la porción que se trasferirá a tubos de Spectronic, para medir la absorbancia a $650 \mu \mathrm{m}$.

A partir de los valores obtenidos, se construyó un diagrama de dispersión, en donde en el eje de las ordenadas (Y) se considera la variable Absorbancia (medida a $650 \mu \mathrm{m}$ ) y en el eje de las abscisas (X) la variable Concentración $(\mathrm{mgC} / \mathrm{mL})$. Se trazó la recta y se estimó el coeficiente de Pearson, a fin de validar la relación lineal entre ambas variables, así como la ecuación de la recta.

Para el análisis de $\mathrm{C}$ en las muestras de suelo, se requirió que éstas, ya secas al aire libre, fuesen cernidas por el tamiz de número 10 (malla de $2 \mathrm{~mm}$ de diámetro), luego se pesó en una balanza analítica de $0.001 \mathrm{~g}$ de precisión, $1 \mathrm{~g}$ de cada muestra de suelo, se llevó la porción a un tubo de ensayo de $20 \mathrm{~mL}$ de capacidad, se le agregó $1 \mathrm{~mL}$ de $\mathrm{H}_{2} \mathrm{O}$ destilada, $2 \mathrm{~mL}$ de la solución de dicromato de potasio y $3 \mathrm{~mL}$ de ácido sulfúrico, se dejó en reposo durante 30 minutos.

Una vez que la temperatura de la solución disminuyó, se le agregó $10 \mathrm{~mL}$ de $\mathrm{H}_{2} \mathrm{O}$ destilada, se agitó en el vortex y luego se centrifugó durante 10 minutos a 5000 r.p.m. La solución sobrenadante fue la empleada para la determinación colorimétrica a la longitud de onda de $650 \mu \mathrm{m}$. Para estimar el \%CO, se procedió bajo la siguiente ecuación:

En donde:

$$
\% \mathrm{CO}=\frac{\text { Concentración de } \mathrm{mgC} / \mathrm{mL}^{*} 100}{\text { Peso seco corregido }(250 \mathrm{~g})}
$$

La Concentración de $\mathrm{mgC} / \mathrm{mL}$, es obtenida a partir de los valores de absorbancia registrados para la muestra de suelo y conociendo la ecuación de la recta obtenida de la curva de calibración, se procede a despejar el valor de X y a sustituir Y por el valor de la absorbancia conocida.

Para determinar el peso seco corregido (250 g), se desarrollaron las siguientes ecuaciones:

1.- Suelo seco $1 \mathrm{~g}=1 / \% \mathrm{H}+1$ (en donde $\% \mathrm{H}=\%$ de humedad previamente conocido).

2.- Suelo seco $0.25 \mathrm{~g}=$ peso de la muestra * suelo seco $1 \mathrm{~g}$. 3.- Suelo seco $250 \mathrm{~g}=$ suelo seco $0.25 \mathrm{~g} * 1000$ 
Para estimar la concentración de carbono en el suelo en toneladas por hectáreas $\left(\mathrm{Mg} \mathrm{ha}^{-1}\right)$ se aplicó la ecuación sugerida por Kauffman et al. (2013): $\% \mathrm{CO}$

$\mathrm{CO}\left(\mathrm{Mg} \mathrm{ha}^{-1}\right)=$ densidad aparente $\left(\mathrm{g} / \mathrm{cm}^{3}\right) *$ intervalo de muestreo*

\section{Determinación de la Materia Orgánica}

Para el porcentaje de Materia Orgánica (\%MO) se empleó el factor convencional de Vammelen (apud Navarro, 2007), lo que supone multiplicar el \%CO en cada caso por 1.724. El resultado, fue cotejado con la escala verbal propuesta por PALMAVEN (1992).

\section{Procesamiento estadístico de datos}

A los fines de precisar el efecto del manglar como fuente de variación en el contenido de $\mathrm{MO}$ y $\mathrm{CO}$ en el suelo, así como la posible incidencia por especie dominante, en comparación con el suelo no rizosférico, se realizó el análisis estadístico a partir del programa IBM SPSS Statistics versión 23.0.

En atención a estos propósitos, los datos se analizaron mediante: (a) ANOVA unifactorial, para determinar el efecto interactivo entre las condiciones, es decir, verificar si las medias de una variable (contenido de MO o C) son diferentes entre los niveles o grupos de otra variable (Cobertura): sin manglar, dominado por $A g$ o dominado por $C e$; y (b) la prueba de diferencia honestamente significativa de Tukey (Tukey's HNR test), para determinar las diferencias significativas entre los grupos de muestras, al comparar el promedio $\mathrm{MO}$ y $\mathrm{C}$, en el suelo sin cobertura de manglar y en el suelo cubierto por cada una de las dos especies en estudio.

\section{Resultados}

\section{Contenido de C en el suelo}

El manglar introduce cambios en el contenido de $\mathrm{C}$ en el suelo. En efecto, las muestras de suelo desprovistos de manglar en su superficie presentaron entre 0.006 y $0.067 \%$ para una media de $0.04 \%$, presentando una desviación estándar de 0.015 , varianza de 0.0023 , evidenciándose una mediana homogeneidad entre los valores correspondientes para las unidades de análisis que constituyen este grupo (Tabla 1).

Las unidades de análisis correspondientes a las muestras de suelo con presencia de Avicennia germinas en superficie, presentaron una desviación 
de 0.016 , varianza de 0.0026 , lo que supone la homogeneidad de las muestras. Estas registraron entre 0.060 y $0.121 \%$ de C, para una media estimada de $0.08 \%$. (Tabla 1). Lo anterior, supone un incremento de hasta $100 \%$, asociado a esta especie de manglar en el aporte de este compuesto orgánico, en contraste con lo referido para el suelo no rizosférico.

Tabla 1. Contenido de C (\%) en el suelo de la Laguna de Unare, según la dominancia en superficie

\begin{tabular}{ccccccc}
\hline \multirow{2}{*}{ Dominancia } & \multicolumn{2}{c}{ \%C } & \multicolumn{3}{c}{ Estadísticos } \\
\cline { 2 - 6 } & mínimo & máximo & X & s & O2 \\
\hline Sin Manglar & 0.006 & 0.067 & 0.04 & 0.015 & 0.0023 \\
$A g$ & 0.060 & 0.121 & 0.08 & 0.016 & 0.0026 \\
Ce & 0.090 & 0.255 & 0.13 & 0.057 & 0.0035 \\
\hline
\end{tabular}

$X=$ media $;=$ desviación típica o estándar; $\mathrm{O} 2=$ varianza

Fuente: Elaboración propia.

Así mismo, se obtuvo para las muestras de suelo con presencia en superficie de Conocarpus erectus, una desviación estándar de 0.057 y varianza de 0.0035 , registrando entre 0.09 y $0.255 \%$ de $C$, para una media estimada de $0.13 \%$ (Tabla 1 ), lo que supone un incremento del $225 \%$ y $62,5 \%$ del aporte al suelo, en comparación con lo registrado para suelos no rizosférico y con presencia de Avicennia germinas, respectivamente.

En la Tabla 2, se presenta el ANOVA estimado por cobertura, presentando un valor $\mathrm{F}$ de 27.643 y significación de 0.000 , lo que permite asumir las diferencias de media de $\mathrm{C}$ entre los grupos de muestras de suelo por cobertura.

Tabla 2. ANOVA del porcentaje de $\mathrm{C}$ en el suelo correspondientes a muestras con superficie desnuda (nr) y dominado por Avicennia germinas (Ag) y Conocarpus erectus (Ce)

\begin{tabular}{ccccc}
\hline & Suma de cuadrados & gl & \multicolumn{1}{c}{$\begin{array}{c}\text { Media } \\
\text { cuadrática }\end{array}$} & F \\
\hline Entre grupos & 0.076 & 2 & 0.038 & 27.643 \\
Dentro de grupos & 0.070 & 51 & 0.001 & \\
Total & 0.146 & 53 & & \\
\hline
\end{tabular}

Fuente: Elaboración propia. 
La prueba HNR Tukey, arrojó una significancia de 0.000 al contrastar los grupos sm-Ag, y sm-Ce, así como 0.008 para el contraste entre Ag-Ce. (Tabla 3)

Tabla 3. Prueba de diferencia honestamente significativa de Tukey para el contenido de MO correspondientes a muestras de suelo no rizosférico (nr) y dominado por Avicennia germinas (Ag) y Conocarpus erectus (Ce)

\begin{tabular}{ccccc}
\hline (I) cobertura & $(\mathbf{J})$ cobertura & Diferencia de medias (I-J) & Desv. Error & Sig. \\
\cline { 2 - 4 } Sin Manglar & AG & $-0.04160^{*}$ & 0.00564 & 0.000 \\
& CE & $-0.09170^{*}$ & 0.01463 & 0.000 \\
\multirow{2}{*}{$\mathrm{Ag}$} & Sin Manglar & $0.04160^{*}$ & 0.00564 & 0.000 \\
& $\mathrm{CE}$ & $-0.05010^{*}$ & 0.01455 & 0.008 \\
$\mathrm{Ce}$ & Sin Manglar & $0.09170^{*}$ & 0.01463 & 0.000 \\
& AG & $0.05010^{*}$ & 0.01455 & 0.008 \\
\hline
\end{tabular}

Fuente: Elaboración propia.

La figura 3, permite evidenciar las diferencias significativas entre los tres grupos de coberturas, permitiendo afirmar que en el sector de Boca de Uchire, el aporte de $\mathrm{C}$ al suelo es mayor en las áreas cubiertas por Conocarpus erectus, que en las dominadas por Avicennia germinans y los suelos desprovistos de vegetación en superficie.

Con base en los resultados anteriores, se estima que para la zona en estudio, la concentración de carbono en el suelo varía de acuerdo a las condiciones de la superficie. En efecto, para el suelo desprovisto de manglar se estimó $0,85 \mathrm{Mg} \mathrm{C} \mathrm{ha}^{-1}$, mientras que en zonas cuya superficie está colonizada por individuos de manglar, esta es significativamente superior al alcanzar 4,41 $\mathrm{Mg} \mathrm{C} \mathrm{ha}^{-1}$, distribuidos en: $1,68 \mathrm{Mg} \mathrm{C} \mathrm{ha}^{-1}$ en áreas con presencia de Avicennia germinas, y $2,73 \mathrm{Mg} \mathrm{C}^{-1}$ en aquellas bajo la influencia del Conocarpus erectus (Figura 4). Para zonas de humedales, se

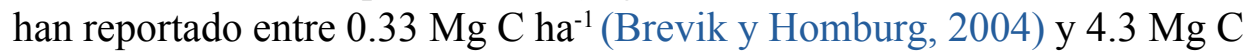
ha-1 (Bernal y Mitsch, 2008). 
Quantification of organic carbon and organic matter in non-rhizospheric soils and covered by Avicennia germinans (L.) and Conocarpus erectus (L.) located in Boca de Uchire, Unare lagoon, State of Anzoátegui, Venezuela.

\section{Figura 3. Porcentaje de $\mathrm{CO}$ en el suelo por grupos de muestras}

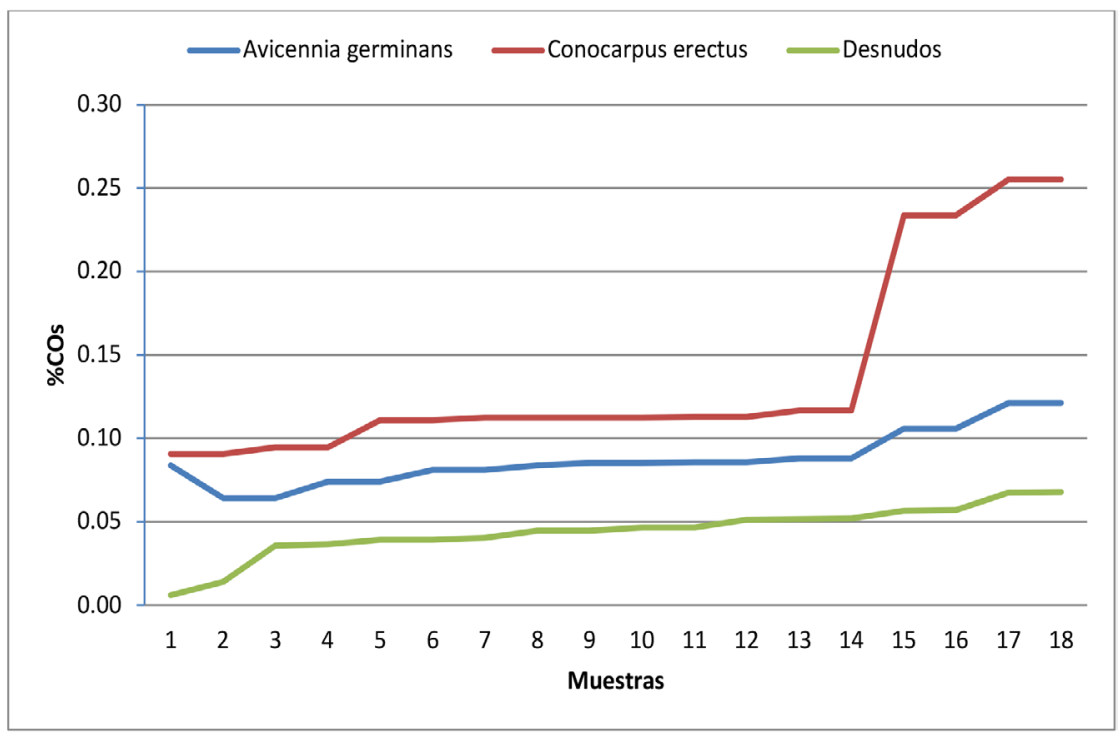

Fuente: Elaboración propia.

Figura 4. Concentración $\mathrm{CO}\left(\mathrm{Mg} \mathrm{ha}^{-1}\right)$ de acuerdo a la cobertura en superficie

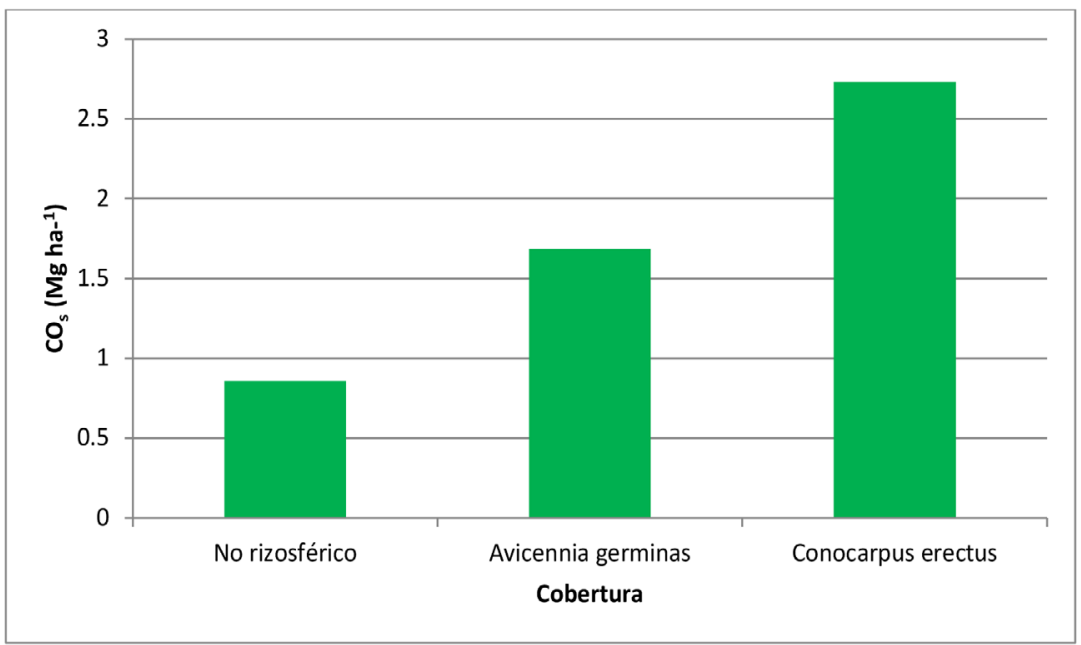

Fuente: Elaboración propia. 
Franklin Núñez-Ravelo María Ugas-Pérez Rossany Calderón-Castellanos

Ferney Rivas-Meriño

Cuantificación del carbono orgánico y materia orgánica en suelos no rizosféricos o cubiertos por Avicennia germinans (L.) y Conocarpus erectus (L.) emplazados en Boca de Uchire, laguna de Unare,

Estado de Anzoátegui, Venezuela.

\section{Contenido de MO (\%)}

El suelo desprovisto de manglar presentó una fracción de MO que oscila entre $0.01 \%$ y $0.11 \%$, para una media estimada de $0.07 \%$. Los datos correspondientes a este grupo presentaron una desviación estándar de 0.02 , y varianza de 0.0007 , lo que permite suponer una mediana homogeneidad entre los valores (Tabla 4).

Tabla 4. Contenido de MO (\%) en el suelo de la Laguna de Unare, según la cobertura en superficie

\begin{tabular}{cccccc}
\hline \multirow{2}{*}{ Dominancia } & \multicolumn{2}{c}{$\%$ MO } & \multicolumn{3}{c}{ Estadísticos } \\
\cline { 2 - 6 } & mínimo & máximo & $\mathbf{X}$ & s & O2 \\
\hline Sin Manglar & 0.01 & 0.11 & 0.07 & 0.026 & 0.0007 \\
$A g$ & 0.11 & 0.20 & 0.15 & 0.026 & 0.0007 \\
Ce. & 0.15 & 0.44 & 0.23 & 0.090 & 0.0100 \\
\hline
\end{tabular}

$X=$ media $;=$ desviación típica $o$ estándar; $\mathrm{O} 2=$ varianza

Fuente: Elaboración propia.

Los suelos con presencia en superficie de Avicennia germinas, registraron un contenido de $\mathrm{MO}$ entre $0.11 \%$ y $0.20 \%$, para un promedio de $0.15 \%$. Este grupo de muestras, arrojó valores de desviación típica y varianza de 0.025 y 0.0007 respectivamente, lo que permite asumir la homogeneidad de los valores obtenidos para las unidades de análisis en este grupo (Tabla 4).

Si bien, este contenido clasifica como muy bajo de acuerdo con lo referido en la escala de PALMAVEN (1992), representa un incremento de aproximadamente $114 \%$ en comparación con el valor promedio estimado para las muestras sin manglar.

Los suelos con presencia de Conocarpus erectus (Tabla 4), presentaron un contenido de $\mathrm{MO}$ entre 0.15 y $0.44 \%$, para una media de $0.23 \%$. El grupo de muestras presenta una desviación típica de 0.09 y varianza de 0.01 , lo que permite suponer una mediana homogeneidad entre los valores obtenidos para las unidades de análisis.

La Tabla 5, muestra que, en el ANOVA, el valor de F es 33.356 y la significación es 0.000 , por lo que al ser la significación menor de 0.05 , se 
asume que las diferencias de media de MO entre los grupos de muestras de suelo por cobertura son significativas.

Tabla 5. ANOVA del contenido de MO correspondientes a muestras de suelo no rizosférico (nr) y dominado por Avicennia germinas (Ag) y Conocarpus erectus (Ce)

\begin{tabular}{cccccc}
\hline Manglares & $\begin{array}{c}\text { Suma de } \\
\text { cuadrados }\end{array}$ & gl & $\begin{array}{c}\text { Media } \\
\text { cuadrática }\end{array}$ & F & Sig. \\
\hline Entre grupos & 0.255 & 2 & 0.127 & 33.565 & 0.000 \\
Dentro de grupos & 0.193 & 51 & 0.004 & & \\
Total & $\mathbf{0 . 4 4 8}$ & $\mathbf{5 3}$ & & \\
\hline
\end{tabular}

Fuente: Elaboración propia.

En efecto, el análisis post hoc indica que diferencias significativas en las comparaciones múltiples realizadas mediante la prueba HNR Tukey, arrojando una significancia de 0.001 al contrastar los grupos sm y Ag, así como 0.000 para el contraste entre sm-Ce y Ag-Ce (Tabla 6).

Tabla 6. Prueba de diferencia honestamente significativa de Tukey para el contenido de MO correspondientes a muestras de suelo no rizosférico (nr) y dominado por Avicennia germinas (Ag) y Conocarpus erectus (Ce)

\begin{tabular}{ccccc}
\hline (I) cobertura & (J) cobertura & $\begin{array}{c}\text { Diferencia de me- } \\
\text { dias (I-J) }\end{array}$ & Desv. Error & Sig. \\
\hline \multirow{2}{*}{$\mathrm{sm}$} & $\mathrm{Ag}$ & $-.07609^{*}$ & .02052 & .001 \\
& $\mathrm{Ce}$ & $-.16792^{*}$ & .02052 & .000 \\
\multirow{2}{*}{$\mathrm{Ag}$} & $\mathrm{Sm}$ & $.07609^{*}$ & .02052 & .001 \\
& $\mathrm{Ce}$ & $-.09183^{*}$ & .02052 & .000 \\
$\mathrm{Ne}$ & $\mathrm{Sm}$ & $.16792^{*}$ & .02052 & .000 \\
& $\mathrm{Ag}$ & $.09183^{*}$ & .02052 & .000 \\
\hline
\end{tabular}

Fuente: Elaboración propia. 


\section{Discusión de resultados}

Los resultados de la investigación confirman la potencialidad forestal de los manglares Avicennia germinas y Conocarpus erectus, para aportar MO al suelo, sino además, en la eficiente captura de $\mathrm{C}$, en contraste con las superficies circundantes, desprovistas de vegetación.

En este sentido, es oportuno referir a Moreno et al. (2002), quienes sostienen que "los manglares constituyen una de las mejores opciones dentro de los humedales costeros, en la captura de carbono, para reducir los efectos que induce la ruptura de la capa atmosférica de ozono y el efecto invernadero sobre el planeta" (p. 116).

En este orden de ideas, Palacios et al. (1990), refieren el aporte de MO del Rizophora sp., y analizan algunos parámetros edafológicos, destacando los porcentajes de MO y de arena, en dicha productividad. Refieren que la producción promedio en peso seco de hojarasca es de $80.2 \mathrm{~g} \mathrm{~m}^{2} \mathrm{mes}$ y de $9.63 \mathrm{Mg} \mathrm{ha}^{-1} \mathrm{año}^{-1}$, siendo que la MO del suelo en general es baja, registrándose el valor más alto en $1.5 \%$ para muestras colectadas a $20 \mathrm{~cm}$ de profundidad.

Donato et al. (2011), afirmaron que estos bosques, constituyen los ecosistemas de mayor contenido de carbono, llegando a almacenar hasta $1023 \mathrm{Mg} \mathrm{C} \mathrm{ha}^{-1}$, por lo que resultan de fundamental interés como espacios estratégicos para la mitigación del incremento de las temperaturas a nivel global.

Ahora bien, Moreno et al. (2010), reportaron diferencias significativas en el almacenaje de carbono en bosques de este tipo, siendo más alto en suelos inundados con vegetación dominante de Rhizophora mangle, en contraste con aquellas parcelas inundadas sólo ocasionalmente, donde la vegetación dominante es el Conocarpus erectus.

En este orden de ideas, Valdés et al. (2011), reportaron diferencias significativas en el aporte de $\mathrm{C}$ por especie de manglar, afirmando que el valor más alto se evidenció para la asociación Laguncularia racemosa Rhizophora mangle, con $106.7 \mathrm{Mg} \mathrm{C}^{-1}$ y más bajo en la de Rhizophora mangle -Laguncularia racemosa - Avicennia germinans, con $73.7 \mathrm{Mg} \mathrm{C}$ $\mathrm{ha}^{-1}$. Sin embargo, consideran que los manglares que más contribuyeron al contenido de $\mathrm{C}$ en el suelo se encontraron en las geoformas de clase orilla, con presencia de Laguncularia racemosa (96.6 $\mathrm{Mg} \mathrm{C} \mathrm{ha}^{-1}$ ) y Rhizophora 
Mangle (81.5 $\mathrm{Mb} \mathrm{C}^{-1}$ ), dejando en una tercera posición a los individuos de Avicennia germinans (81.4 $\mathrm{Mg} \mathrm{C}^{\mathrm{a}}{ }^{-1}$ ).

Con base en los resultados obtenidos, así como los reportados en los trabajos de Moreno et al. (2010) y Valdés et al. (2011), se puede inferir que si bien la cobertura constituye un elemento modificador en el aporte de $\mathrm{C}$ al suelo, en el caso de los bosques de manglar, cada especie contribuye de manera particular con su potencialidad forestal, en el aporte y captura del referido elemento.

En este sentido, se puede inferir que, considerando la especie de manglar en superficie, la Laguncularia racemosa, presenta el mayor aporte de carbono orgánico al suelo, seguido por el Rhizophora mangle, Conocarpus erectus y Avicennia germinans.

De allí que los manglares emplazados en Boca de Uchire, si bien aportan significativamente carbono orgánico al suelo, es posible que por estar constituido por individuos de las especies Conocarpus erectus y Avicennia germinas, y siendo estos los generan un menor aporte de $\mathrm{CO}$, el sector experimente una menor concentración que la reportadas para otros ecosistemas de manglar de mayor complejidad biodiversa en el mundo, o incluso, en aquellos bosques constituidos por las mismas especies. Considerando que en este caso, como ya hemos referido, se trata de un boscaje relativamente joven, cuyos individuos se encuentran en cobertura abierta o dispersa.

Ahora bien, es importante destacar que en cuanto a las potencialidades forestales de la Avicennia germinans, Jiménez y Lugo (ob.cit), reportaron que se trata de una especie que produce rápidamente cantidades importante de hojarasca, haciendo énfasis que la materia orgánica se lixivia de las hojas de mangle con facilidad, y posee una relación de carbono a nitrógeno baja, que resulta en unas altas tasas de descomposición.

De allí que el aporte de MO y C en suelos cubiertos por Avicennia germinans, en comparación con los desprovisto de especies arbóreas, genere condiciones para la proliferación de ciertos microorganismos, lo cual tiene efectos positivos sobre esta especie de manglar, ya que de acuerdo con lo referido por Lovelock et al. (2016), contribuye con un posible aumento en la productividad del nivel de la hoja, así como en su índice del área, considerando que esto último pudiese compensar la reducción en el uso de agua por hojas individuales. 
Núñez y Ugas (2019), reportaron para Boca de Uchire una diferencial emisión de $\mathrm{CO}_{2}$ en el suelo por cobertura, afirmando que aquellos que se presentan desprovistos de vegetación en superficie, produjeron $3,74 \mathrm{mg}$ $\mathrm{C}-\mathrm{CO}_{2} \mathrm{~g} / 24 \mathrm{~h}$, mientras que los dominados por manglar arrojaron $12,25 \mathrm{mg}$ $\mathrm{C}-\mathrm{CO}_{2} \mathrm{~g} / 24 \mathrm{~h}$, estimando para la superficie cubierta por Avicennia germinas $10,61 \mathrm{mg} \mathrm{C}-\mathrm{CO}_{2} \mathrm{~g} / 24 \mathrm{~h}$.

Esto evidencia la potencialidad forestal de los manglares, no solo para generar condiciones que favorecen la proliferación de microorganismos, y con ello la producción de $\mathrm{CO}_{2}$ asociado a su respiración sino, además, su captura en la estructura del suelo, convirtiéndolos en sumideros de este gas.

En los suelos con presencia de Conocarpus erectus en el área de estudio, como ya se refirió, evidenciaron la mayor fracción de $\mathrm{MO}$ y C en comparación con los suelos con presencia de Avicennia germinas, así como aquellos que no presentan manglar en su superficie.

Lo anterior, guarda correspondencia con lo referido por Aceituno et al. (2016), quienes sostienen que los manglares en Guatemala, evidencian para los bosques con presencia de la especie de Conocarpus erectus, una densidad de CO superior a los que presentan Avicennia germinans.

Ahora bien, la efectividad del Conocarpus erectus para generar condiciones que favorezcan la concentración de $\mathrm{MO}$ y $\mathrm{C}$ en el suelo que ocupan, no ha sido suficientemente estudiada. No obstante, Von Carlowitz et al. (1991), reportaron que esta especie de manglar es muy rica en contenido de MO, advirtiendo que puede crecer bajo condiciones de inundación permanente o estacional en sitios con salinidad fuerte y moderada.

Adicionalmente, se reconoce que las condiciones edáficas constituyen otro elemento interviniente en las potencialidades de este para la captura de carbono. En efecto, existen reportes que vinculan los órdenes del suelo con su potencialidad de almacenamiento. De allí que Martínez et al. (2008), refieran que entre los que acumulan la mayor cantidad de este elemento, se encuentran los del orden Histosols, pese a que suelen tener la menor superficie con respecto a otros órdenes, que presentan la mayor relación cantidad $\mathrm{CO} /$ superficie.

Eswaran et al. 1993 (citado en Martínez et al., 2008), precisaron una jerarquización del aporte de carbono de acuerdo al orden de suelo, indicando que de mayor a menor capacidad de captura, se ubican: Histosols, 
Andisols, Inceptisols, Spodosols, Mollisols, Oxisols, Entisols, Ultisols, Alfisols, Vertisols y Aridisols.

A modo de referencia, se puede considerar para Histosols entre 470 y $820 \mathrm{Mg} \mathrm{C}^{-1}$ (Moreno et al., 2002), Oxisols entre 120 y $240 \mathrm{Mg} \mathrm{C}^{-1}$ (Lal, 2001), y Vertisols 69.9 Mg C ha'-1 (García et al., 2006).

Considerando que el suelo en Boca de Uchire corresponde al orden Vertisols, se puede afirmar que esta condición constituye otro elemento que limita su potencialidad de captura de $\mathrm{C}$, en relación con los reportados para otras zonas del mundo con cobertura de las referidas especies.

En efecto, de acuerdo con García et al. (2006), los valores más bajos en este orden, se deben al escaso desarrollo de su estructura, en donde el $\mathrm{C}$ al ser liberado de los agregados, queda fácilmente disponible para los microorganismos.

\section{Conclusiones}

La investigación evidenció que el contenido de $\mathrm{CO}$ y $\mathrm{MO}$ en el suelo, al inicio de la estación lluviosa, presenta diferencias significativas atendiendo a la modificación que se introduce a partir de la cobertura superficial.

Los suelos colonizados en superficie por alguna de las especies de manglar estudiadas, aun cuando registraron bajo contenido de $\mathrm{CO}$ y $\mathrm{MO}$, este resultó significativamente superior a lo estimado para los suelos desprovistos de la referida cobertura.

En efecto, se estimó que el aporte del $\mathrm{CO}$ y $\mathrm{MO}$ en las superficies colonizadas por Avicennia germinas y Conocarpus erectus, es dos y tres veces superior a lo evidenciado para el suelo no rizosférico.

$\mathrm{Al}$ analizar las variaciones introducidas por la dominancia en superficie de las especies en estudio, se evidenció que los suelos colonizados por Conocarpus erectus, presentan una mayor fracción de $\mathrm{CO}$ y $\mathrm{MO}$ en comparación con aquellos cuya superficie está cubierta por Avicennia germinas, lo que indica una mayor potencialidad forestal de la referida especie en el aporte de estos elementos.

Tal variación puede estar atribuida a las características fisionómicas y fisiológicas propias de cada especie, ya que como se refirió, el Conocarpus erectus por sus rasgos estructurales, presenta un importante contenido de MO. 
Ahora bien, es posible que la juventud del manglar de Boca de Uchire, las condiciones físicas y químicas propias del suelo en donde este se desarrolla, aunado a los efectos vinculados a la intervención antropogénica, constituyan condiciones que limitan la potencialidad de este bosque en la captura de $\mathrm{C}$ y aporte de $\mathrm{MO}$, en relación con los reportados para otras zonas del mundo.

Sin embargo, con base en los hallazgos de la presente investigación, se puede concluir que: (a) el incremento de la MO en el suelo, inducido por las condiciones edáficas que genera la presencia de la Avicennia germinans y el Conocarpus erectus, no solamente es un indicador del potencial de estas especies para la generación, almacenamiento y transformación del carbono, sino que además permite inferir su aporte en la evolución edáfica, ya que posiblemente esté incidiendo en la CIC, favoreciendo la agregación de las partículas, así como propiciando condiciones para el desarrollo de la actividad microbiana; y (b) se reafirma que los ecosistemas de manglar, contribuyen como reguladores en el flujo del carbono, y su captura en la estructura del suelo.

Finalmente, se considera necesario continuar con la investigación del manglar de Boca de Uchire, a fin de precisar: (a) si la potencialidad atribuida al Conocarpus erectus varía en función de la estacionalidad de las lluvias; (b) se mantiene el contenido de $\mathrm{CO}$ y $\mathrm{MO}$ a mayor profundidad del perfil del suelo, y (c) cuantificar la reserva total de carbono de este bosque, a los fines de comprender su aporte en el flujo del referido gas invernadero.

\section{Referencias}

Aceituno, M., Cifuentes, C., Cojom, J. y Bosarreyes, A. (2016). Estimación del contenido de carbono en el componente arbóreo del ecosistema manglar en el pacífico de Guatemala. Recuperado de: http://201.207.189.89/bitstream/handle/11554/8734/Estimacion_ del_contenido_de_carbono.pdf?sequence=1\&isAllowed=y

Alef, K. (1995). Soil respiration. En: Alef. K and P. Nannipieri (eds). Methods in applied soil microbiology and biochemistry. USA San Diego: Academic Press Limited, 214-218. doi: 10.1016/ B978-012513840-6/50025-2 
Barboza, F., Barreto, M., Figueroa, V., Francisco, M., González, A., Lucena, L., Mata, K., Narváez, E., Ochoa, E., Parra, L., Romero, D., Sánchez, J., Soto, M., Vera, A., Villarreal, A., Yabroudi, S. y Medina, E. (2006). Desarrollo estructural y relaciones nutricionales de un manglar ribereño bajo clima semiárido. Ecotrópicos 19(1), 13-29.

Bernal, B. y Mitsch, W. (2008). A comparison of soil carbon pools and profiles in wetlands in Costa Rica and Ohio. Ecological Engineering. 34, 311-323. Recuperado de: https://www.academia. edu/17933657/A_comparison_of_soil_carbon_pools__ and profiles_in_wetlands_in_COta_Rica_and_Ohio

Brevik, E. y Homburg, J. (2004). A 500 year500-year record of carbon sequestration from a coastal lagoon and wetland complex, Southern California, USA. Catena 57, 221-232. Recuperado de: https://www.sciencedirect.com/science/article/abs/pii/S03418 16203002613?via\%3Dihub

Casanova, E. (2005). Introducción a la ciencia del suelo. Caracas: UCVConsejo de desarrollo Científico y Humanístico.

Cumana, L. Prieto, A. y Ojeda, G. (2000). Florula de la laguna de Chacopata, Península de Araya, estado Sucre, Venezuela. Revista Saber 12, 25-33.

De Olivares, D. (1988). “Caracterización ecológica del manglar de la Ciénaga Las Palmitas" (estado Zulia. Venezuela). Boletín del Centro de Investigaciones Biológicas 17, 91- 119

Del Mónaco, C., Giménez, E., Narciso, S., Alfonso, F. y Bustillos, F. (2010). "Caracterización de los bosques de manglar y las praderas de Thalassia testudinum de la Isla La Tortuga y Cayos adyacentes, Venezuela." Boletín del Centro de Investigaciones Biológicas 44 (3), 297-316.

Donato, D., Boone, J., Murdiyarso, D., Kurnianto, S., Stidham, M. and Kanninen, M. (2011). Mangroves among the most carbon rich forests in the tropics. Nature Geoscience, 293-297. doi: 10.1038/ngeo1123

Duhne, M. (2011). Los manglares, ecosistemas claves. ¿Cómo ves? Revista de divulgación de la ciencia de la Universidad Nacional Autónoma de México. Recuperado de: http://www.comoves.unam.mx/ numeros/indice/5 
Elizalde, G., Viloria, J. y Rosales, A. (2007). Geografía de los suelos de Venezuela. GeoVenezuela 2. Caracas: Fundación Empresas Polar.

García, M., Alliaume, F., Mancassola, V. y Dogliotti, S. (2011). Carbono orgánico y propiedades físicas del suelo en predios hortícolas del sur de Uruguay. Agrociencia Uruguay 15,1. Recuperdado de: http://www.fagro.edu.uy/agrociencia/index.php/directorio/article/ view/97/55

García, R., Espinosa, D., Figueroa, B., García, N. y Gallardo, J., (2006). Reservas de carbono orgánico y de fracciones húmicas en un Vertisol sometido a siembra directa. Terra latinoamericana, 24 (2), 241-251.

Gros, A. y Domínguez, A. (1992). Abonos guía práctica de fertilización. Madrid: Ediciones Mundi-Prensa.

Ibáñez, J. (2006). Humus y la Clasificación del Humus de los Suelos. Recuperado: http:// www.madrimanr.org/blogs/universo/2006/08/18/

Jiménez, J. y Lugo, A. (s.f). Avicennia germinans (L.) L. SOITF-SM-4. New Orleans: U.S. Department of Agriculture, Forest Service, Southern Forest Experiment Station. Recuperado: http://www.fs.fed. us/global/iitf/Avicenniagerminans.pdf

Kauffman, J., Donato, D. y Adame, M. (2013). Protocolo para la medición, monitoreo y reporte de la estructura, biomasa y reservas de carbono de los manglares. ,. Indonesia: CIFOR.

Lal, R. (2001). World cropland soils as a source or sink for atmospheric carbon. Advances in agronomy, 71, 145-191.

Lara, S., Suarez, C. y Marcucci, E. (1997). Atlas morfodinámico costero de Venezuela. Caracas: Universidad Pedagógica Experimental Libertador.

López, B., Barreto, M. y Conde, J. (2011). Caracterización de los manglares de zonas semiáridas en el Noroccidenteel Noroccidente de Venezuela. Interciencia 36 (12), 888-893.

Lovelock, C., Krauss, K., Osland, M., Reef, R. and Ball, M. (2016). The physiology of Mangrove Trees with Changing climate (149-180) En: Goldstein, G. y L. Santiago (ed.) Tropical Tree Physiology. Adaptations and Responses in a Changing Environment. Springer, FloridaUSA. doi: 10.1007/978-3-319-27422-5_7 
Martínez, E. Fuentes, J. y Acevedo, E. (2008). Carbono orgánico y propiedades del suelo. Revista de la ciencia del suelo y nutrición vegetal 8(1), 68-96. https://scielo.conicyt.cl/pdf/rcsuelo/v8n1/art06.pdf

Mattia, M. y Méndez, D. (2003). Inventario de los aprovechamientos hidráulicos de la Cuenca del río Unare. Casos en estudio: embalses "el Cuji" y "el andino". (Trabajo de Grado). Escuela de Ingeniería. Universidad Central de Venezuela, p.153.

Medina, E. y Barboza, F. (2003). Manglares del sistema del Lago de Maracaibo: Caracterización fisiográfica y ecológica. EcotropicosEcotrópicos 16(2), 75-82.

Méndez, J. (2007). Costas, litorales del Caribe y del Atlántico, Islas y archipiélagos: Las profundidades marinas. GeoVenezuela 2. Caracas: Fundación Empresas Polar.

Moreno, E. Guerrero, A. Gutiérrez, M. Ortiz, D y Palma, D. (2002). Los manglares de Tabasco, una reserva natural de carbono. Madera y Bosques. 8 (1), 115-:128. Recuperado: http://www.inecol.edu.mx/ myb/resumeness/no.\%20esp.1/pdf/emoren\%20et\%20al\%202002. pdf.

Moreno, G., Cerón, J., Cerón, R., Guerra, J., Amador, L. y Endañú, E. (2010). Estimación del potencial de captura de carbono en suelos de manglar de Isla del Carmen. UNACAR Tecnociencia. Recuperado: http://www.unacar.mx/contenido/tecnocien cia/tecnociencia6/ tema_3_estimacion_del_potencial.pdf

Navarro, J. (2007). Variación del Contenido de Materia Orgánica de suelos volcánicos sometidos a distintos manejos agrícolas. (Tesis de Grado no publicada). Universidad Austral de Chile, Facultad de Ciencias Agrarias, Escuela de Agronomía. Recuperado: http://cybertesis.uach.cl/tesis/uach/2007/fan936v/doc/fan 936v.pdf

Navarro, J., Moral, R., Gómez, I. y Mataix, J. (1995). Residuos orgánicos $y$ agricultura. Alicante: Universidad de Alicante

Núñez, F. y Ugas, M. (2018). Caracterización fisionómica del Manglar de Avicennia germinans y Conocarpus erectus emplazados en la Laguna de Unare, Venezuela. Terra. Nueva etapa. XXXIV (55), 193-218. Núñez, F. y Ugas, M. (2019). Flujo de $\mathrm{CO}_{2}$ en suelos dominados por Avicennia germinas y Conocarpus erectus. Revista Geográfica Venezolana. 60(2), 284-299. 
Ojeda, M. (2004). Informe Técnico. Proyecto Evaluación de las poblaciones de patos migratorios (Anatidae) en Venezuela, 2003-2004. Recuperado: https://n9.cl/jz1j

Organización de las Naciones Unidas para la Alimentación y la Agricultura -FAO- (2001). Tendencia general de la captura de Carbono en el suelo. En: FAO: Captura de carbono en los suelos para un mejor manejo de la tierra. Recuperado: http://ftp.fao.org/ docrep/fao/004/ y2779S/y2779s00.pdf

Organización de las Naciones Unidas para la Alimentación y la Agricultura -FAO- (2017). Propiedades químicas del suelo: Carbono orgánico. [Información en línea]. En: FAO: Portal de suelos de la FAO. Recuperado: http://www.fao.org/soils-portal/soil-survey/ propiedades-del-suelo/propiedades-quimicas/es/

Palacios, M., Vargas, E. y De La Pava, M. (1990). Determinación del aporte de materia orgánica del Manglar en la zona de Bocagrande. Boletín Cientifico CCCP. 1, 55-72.

PALMAVEN, Filial de Petróleos de Venezuela (1992). Análisis de suelo y su interpretación, Serie Técnica, publicaciones de divulgación científica, Caracas: Autor.

Pannier F. y Pannier, R. (1989). Los Manglares de Venezuela. Serie Cuadernos Lagoven, Lagoven SA, Caracas, Venezuela.

Peris, M. (2013). El suelo como sumidero de $\mathrm{CO}_{2}$ : mapa de retención de $\mathrm{CO}_{2}$ de las tierras de labor de los distintos países de la Unión Europea. Ojeando la Agenda 24: s/p

Ramos, E. y Zuñiga, D. (2008). Efecto de la humedad, temperatura y $\mathrm{pH}$ del suelo en la actividad microbiana a nivel de laboratorio. Ecología aplicada 7 (1,2), 123-130. doi: 10.21704/rea.v7i1-2.367

Roa, P. (1990). La transgresión Flamenca y la evolución de la laguna de Unare, Venezuela. Boletín del Instituto de Oceanografía-Universidad de Oriente 29 (1/2): 11-25.

Robinson, G. (1967). Los suelos. Su origen, constitución y clasificación. Barcelona: Ediciones Omega.

Rodríguez, C., Villamizar, E. y Rodríguez, J. (2009). Caracterización de la Comunidad Bentónica en la Laguna de Unare (Venezuela). VI Congreso de Investigación. Universidad de Carabobo. Recuperado: https://n9.cl/jtoz 
Rodríguez, J. y González, D. (2001). Estudio ambiental de la cuenca del río Unare y las lagunas de Unare y Píritu. Caracas: Universidad Central de Venezuela.

Rodríguez, M. y Gómez, I. (2016). Actividad microbiana en sedimentos superficiales del Bosque de manglar (Rhizophora mangle) de la bahía de Pertigalete (Anzoátegui, Venezuela), durante los períodos de Surgencia y transición. Saber 29 (3), 511-522

Sainz, H., Echeverría, H. y Angelini, H. (2011). Niveles de carbono orgánico y $\mathrm{pH}$ en suelos agrícolas de las regiones Pampeana y Extrapampeana Argentina. Ciencia del Suelo. 29. Recuperado: http:// www.scielo.org.ar/scielo.php?Script=sci_ar ttextypid $=\mathrm{S} 1850$ 20672011000100004

Sánchez, L., Paolini, J. y Rodríguez, J. (2010). Dinámica de las propiedades del suelo en bosques de Rhizophora mangle L. (Rhizophoraceae) en Isla de Margarita, Venezuela. Revista de Biología Tropical 58 (2), 547-564. doi: 10.15517/rbt.vrbt. v58i2.5228

Sebastiani, M., Moreno, M., Soto, A., Aguirre, E., Camacho, L., Medina, R., Yranzo, A., y Zamora, A. (2007). Propuesta para la evaluación ambiental estratégica de zonas especiales de desarrollo sustentable (ZEDES): Cuenca del río Unare, estados Anzoátegui y Guárico-Venezuela. Revista Geográfica Venezolana. 48 (1). Recuperado: https:// www.redalyc.org/pdf/3477/347730365003.pdf

Segura, M., Sánchez, P., Ortiz, C. y Gutiérrez, M. (2004). Carbono orgánico de los suelos de México. Terra Latinoamericana, 23 (1), 21-2.

Senior, W. y Ocando, L. (s/f). Estudio hidroquímico de la Laguna de Unare, Venezuela, durante el periodo noviembre 1988-julio 1989. Recuperado: https://www.researchgate.net/profile/William_Senior4/ publication/307925816_Informe_sobre_la_Laguna_de_Unare/ links/57d1e8aa08ae0c0081e05810.pdf

Suárez, C. (1991). Modificaciones naturales y artificiales en las lagunas de Unare y Píritu y sus efectos ambientales: Un enfoque geomorfológico. Universidad Pedagógica Experimental Libertador, Instituto Pedagógico de Caracas. Caracas, Venezuela. Trabajo de Ascenso (inédito). 
Swift, R. (2001). Sequestration of Carbon by Soil. Soil Science,166, 858871. Recuperado: http://dx.doi.org/10.1097/00010694-20011100000010.doi:10.1097/00010694-2001 11000-00010

Toledo, V. (2008). Determinación de Carbono Orgánico en muestras de Suelos por la técnica colorimétrica, en el método de combustión húmeda de Walkley - Black. (Trabajo no Publicado). Línea de Investigación Ambientes Áridos y Semiáridos en Venezuela, UPEL - IPC. United States Department of Agriculture (1999). Soil Taxonomy A Basic System of Soil Classification for Making and Interpreting Soil Surveys. Washington: Superintendent of Documents, U.S. Government Printing Office. Recuperado: https://www.nrcs. unra.gov/Internet/ FSE_DOCUMENTS/nrcs 142p2_051232.pdf

Valdés, E., Valdez, J., Ordaz, V., Gallardo, J., Pérez, J. y Ayala, C. (2011). Evaluación del carbono orgánico en suelos de los manglares de Nayarit. Revista Mexicana de Ciencias forestales. Recuperado: http://www. scielo.org.mx/scielo.php?script $=$ sci_ arttextypid=S2007-11322011 000600005

Velasco, P. (1989). Humus y evolución regresiva de los suelos. Ecología. Recuperado: http://www.mapama.gob.es/es/parquesnacionalesoapn/ publicaciones/ecología_03_04_tcm7-458 07.pdf

Vivas, L. (2012). Geotemas. Mérida: Fundación Fondo Editorial Simón Rodríguez.

Von Carlowitz, P., Wolf, G., y Kemperman, R., (1991). The Multipurpose and Shrub Database. An Information and Decision-Support System. Manual, Version 1.0. Nairobi-Kenia: ICRAF 
